Article

\title{
Use of Blockchain Technology to Curb Novel Coronavirus Disease (COVID-19) Transmission
}

\author{
Asma Khatoon \\ Department of Electrical \& Electronic Engineering, National University of Ireland, Galway H91 TK33, Ireland; \\ a.khatoon1@nuigalway.ie
}

\begin{abstract}
WHO was informed on 31 December 2019 of cases of unknown cause pneumonia in Wuhan City, China. On 7 January 2020 Chinese authorities reported a novel coronavirus as the cause and was temporarily labeled "2019-nCoV." Coronaviruses (CoV) are a wide family of viruses which cause diseases ranging from common cold to more serious illnesses. A novel coronavirus (nCoV) is a new strain not previously found in humans. Countries around the globe have stepped up their surveillance to quickly detect any new 2019-nCoV cases. Blockchain is developing into a safe and efficient network for secure data sharing in applications such as the financial industry, operations management, food industry, energy market, the Internet of Things and healthcare. In this paper, we are using blockchain technology as a mean to share authentic data, tracking of relevant information and help speed up the treatment process. At the same time it will preserve person's identity. Timely deployment and suitable implementation of the proposed model have the opportunity to curb COVID-19 transmissions and associated mortality, especially in environments with inadequate access to testing facilities. This work will also facilitate in the treatment of other infectious diseases. Smart contract have been designed and implemented using the ethereum blockchain platform which has been presented in this paper. This work would facilitate multiple stakeholders who are involved within the medical system to curb the transmission of this disease.
\end{abstract}

Keywords: Coronaviruses; Blockchain Technology; COVID-19; Smart Contracts; Data Exchange; Secure; Distributed Ledger Technology; Healthcare

\section{Introduction}

The COVID-19 coronavirus is currently affecting 208 countries and regions worldwide, as well as 2 international ships [1]. To determine the extent of the risk posed by this novel coronavirus, there are three criteria to be understood: Transmission rate (Ro) - number of newly infected individuals from a single case, Case Mortality rate (CMR) - frequency of death cases and assessment whether asymptomatic transmitting is possible. A virus 'attack rate or transmissibility (how quickly the disease spreads) is indicated by its reproduction number (Ro, pronounced as R-nought or r-zero), which indicates the actual number of people to whom the virus can be transmitted by a single infected individual. WHO predicts (on Jan. 23) that Ro would be between 1.4 and 2.5. Other research measured Ro to be somewhere between 3.6 and 4.0, and 2.24 to Ro had estimated the preliminary studies to be between 1.5 and 3.5. An epidemic with a reproduction number below 1 slowly vanishes. For contrast, the Ro is 1.3 for common flu and 2.0 for SARS [2].

\section{COVID-19 and Blockchain Technology}

Blockchain technology has recently emerged as a key technology in the healthcare sector's digital transformation and many research studies [3]-[8], [9]-[15] have identified potential for blockchain to the healthcare ecosystem. The manner in which mainstream medical systems and companies have been engaged in the healthcare sector over the last few decades is ready to transform. 
Information and communication technologies (ICTs) and blockchain are key technologies that allow healthcare institutions to be decentralized and digitized, and provide patients as well as service providers with modern and digitized healthcare ecosystems [16], [17], [26]-[31], [18]-[25]. Blockchain data management systems build services for patients, physicians, and healthcare institutes in the fields of patient record access and monitoring, claims and payment processing, medical IoT protection management, and clinical data verification and financial audit exchange, and transparency. Real-time updates to an encrypted, anonymous blockchain database are done in these applications to understand, track, and manage medical information. It also enables restricting the unauthorized person's access to confidential information by health care organizations.

Management of healthcare during this COVID-19 crisis and in general includes several processes such as financial management, personnel, patients, legal problems, logistics, inventory, etc [32]. Medical workflows include routine activities related to the treatment of patients, which can be depicted as a sequence of conditional acts. These are designed to provide stronger internal controls, improve quality, compliance, profitability, and reduce risk, work cycles and overhead in hospitals and other providers of health care. In this paper, we have introduced ethereum based solution to prevent transmission of COVID-19. This also concerns personal data protection, the processing of confidential information, the removal of third parties, identity management. Unlike centralized networks, the functionality of the network continues even though single nodes break down. It improves confidence, because individuals do not assess the trustworthiness of the intermediary or other network participants. When people develop trust within the program itself, it is enough. The lack of intermediaries also facilitates the data protection [33]. As in the existing practice of third parties gathering personal data, there is the risk of security breaches. By using the blockchain third parties can become redundant, effectively increasing user security. Through using the blockchain, third parties can become redundant, effectively increasing user security. At the same time it can preserve person's identity. On 30 Jan 2020, the World Health Organization declared the outbreak of coronavirus a global emergency for public health [34].This paper aims to suggest the possible usage of blockchain in the treatment of COVID-19. Prompt deployment and proper implementation of the proposed model would have the potential to curb COVID-19 transmissions and related mortality, especially in environments with insufficient access to test facilities. The ethereum blockchain technology introduced in this paper has been used to develop and execute smart contracts. This research will promote many actors interested in curbing the spread of this disease within the medical system.

\section{System design and implementation}

The implementation of the platform would be a decentralized application (DApp) supporting a private blockchain network with a distributed file system (DFS) at the back end. Ethereum was used to introduce smart contract framework for healthcare blockchain. This is an open source platform and currently one of the biggest public blockchain networks with an established community and a large collection of public DApps. The platform currently uses a consensus proof-of-work (PoW) algorithm called Ethash, but engineers are working to turn it into a proof-of-stake (PoS) scalability algorithm in the immediate future. Ideally, for the design of distributed applications, a Delegated Proof-of-Stake (DPoS) or Functional Byzantine Fault Tolerance (PBFT) consensus algorithm is suited. The DApp will have the potential to detect irregularities, unauthorized data insertions and missing entities by matching DFS content with ledger registers. Each phase is labelled with an Audit Timeline. The essential elements of the smart contracts are functions, events, state variables, and modifiers and are written in the solidity programming language. To pay the transaction fee, Remix and Kovan test network is used to deploy smart contracts on the testnet and testnet ethers. Three stages are involved in the development of smart contracts, which use Solidity programming to write, compile, and announce. The bytecode is created by the real time compiler Solidity. Ethereum Wallet has been used 
to disclose smart Blockchain contracts. Since smart contract programming began with Ethereum and Solidity, it is still a discipline under growth. Ethereum uses a particular elliptic curve and set of mathematical constants as defined by the US National Institute of Standards and Technology (NIST) standard called secp256k1. Elliptic curve cryptography, or ECC, is a strong technique to cryptography from a very well-known RSA, and an evolutionary method. By u sing the mathematics behind elliptic curves to establish privacy between key pairs is a technique used for public key encryption. Throughout the past few years, ECC has steadily increased in popularity because of its potential to provide the same level of protection as RSA with a much lower key size. The following function describes the secp256k1 curve, which generates an elliptic curve:

Or

$$
p_{2}=\left(q_{3}+7\right) \text { over }(F x)
$$

$$
p_{2} \bmod p=\left(q_{3}+7\right) \bmod x \quad \text { Eq. }(2)
$$

The mod $\mathrm{p}$ (prime number $\mathrm{p}$ modulo) shows that this curve reaches a finite prime order $\mathrm{p}$ field, also written as $\mathrm{F} \mathrm{x}$, where $x=2^{256}-2^{32}-2^{9}-2^{8}-2^{7}-2^{6}-2^{4}-1$, that's a very large number. Because this curve is defined over a finite prime order field rather than over the real numbers, it looks like a two-dimensional pattern of dots, making it difficult to imagine. Choosing a group ' $\mathrm{P}$ ' with a very large group cardinality or number, connoted by \# $Q$ and a large ' $r$ ' is important, certainly in a cryptographic sense. When operating in the spatial plane, on any smooth cubic curve, we can define a group structure. In the normal form of Weierstrass, such a curve will have an additional point at infinity, $\mathrm{O}$, at the homogeneous coordinates that function as the group's identity.

Since the curve is symmetrical about the $\mathrm{X}$-axis, we can take $-\mathrm{X}$ to be the opposite point, provided any point $X$. We're considering $-\mathrm{O}$ to be $\mathrm{O}$.

If $X$ and $Y$ are two points on the curve, then in the following way we can describe uniquely a third point, $X+Y$ First, draw the line between $X$ and $Y$. At a third point, $Z$, this will normally intersect the cubic. So we take $X+Y$ as $-Z$, the opposite value to $Z$ as shown in Figure 1 and Figure 2 . 


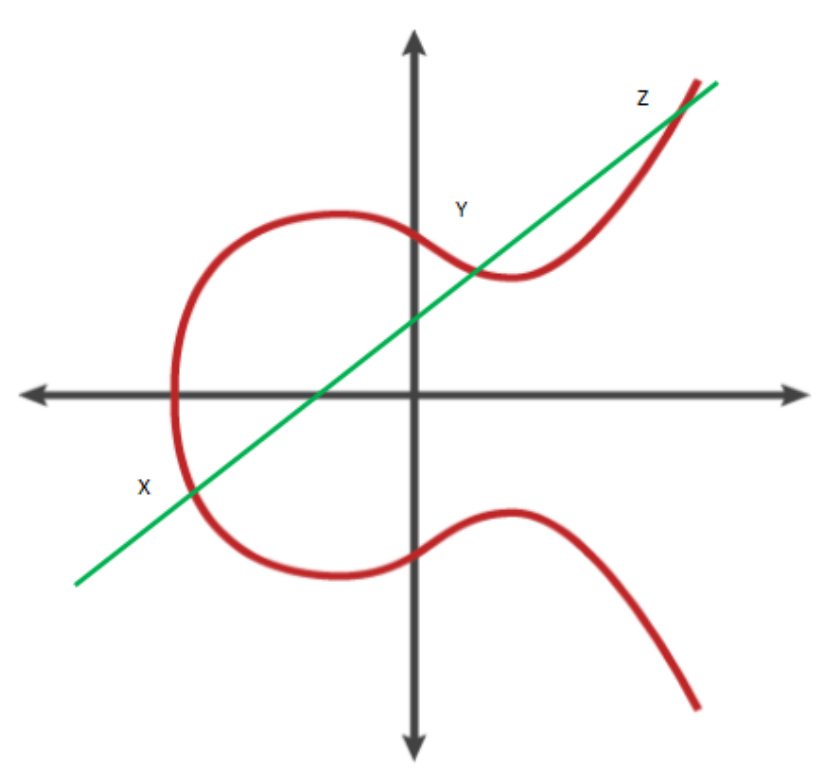

Figure 1. Graph of curves $X+Y+Z=0$

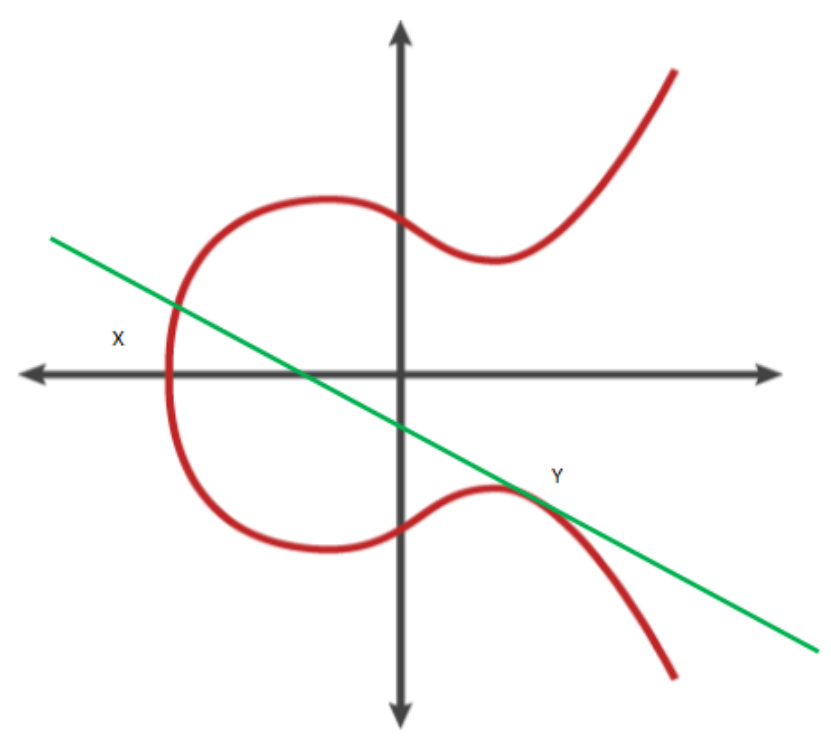

Figure 2. Graph of curves $X+Y+Y=0$

This definition for addition works except for multiplicity of infinity and intersection in a few special cases related to the point. The first point is when $\mathrm{O}$ is one of the points. Here we define the group's identity as $X+O=X=O+X$. First, we define $X+Y=O$ if $X$ and $Y$ are opposite to each other. Ultimately, if we have only one point in $X=Y$, we cannot describe the distance between them. In this case, at this point we are using the tangent line to the curve as our line. The tangent intersects a second point $\mathrm{Z}$ in most situations and we can take the opposite.

We can still describe a group structure for a cubic curve that is not normal in Weierstrass by designating one of its nine inflection points as identity $\mathrm{O}$. In the projective plane, when accounting for multiplicity, the line can intersect a cubic at three points. $-X$ is defined as the unique third point on the line that passes through $\mathrm{O}$ and $\mathrm{X}$ for a point $\mathrm{X}$. Therefore, $\mathrm{X}+\mathrm{Z}$ is defined as $-\mathrm{R}$ for any $X$ and $Y$, where $Z$ is the unique third point on the $X$ and $Y$ column. 
Let $\mathrm{L}$ be a field that determines the curve (i.e. the coefficients of the defining equation or curve equations are in L) and denote the curve by M. So M's L-rational points are the points on M, the coordinates of which are all in $\mathrm{L}$, including the infinity point $\mathrm{M}(\mathrm{L})$ denotes the set of L-rational points. It also forms a group, since polynomial equation properties show that if $X$ is in $M(L)$, then $-X$ is also in $M(L)$, and if two of $X, Y$, and $Z$ are in $M(L)$, then the third is the same. Therefore, if $L$ is a $\mathrm{K}$ subfield, then $\mathrm{M}(\mathrm{L})$ is an $\mathrm{M}(\mathrm{K})$ subgroup. Graph of curves are illustrated in Figure 3 and Figure 4.

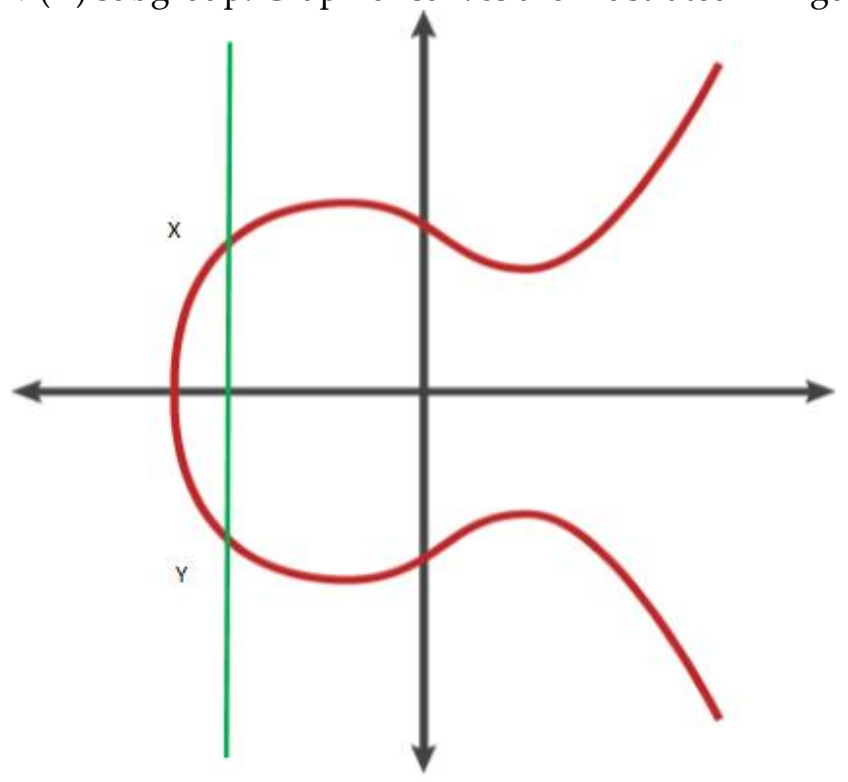

Figure 3. Graph of curves $X+Y+0=0$

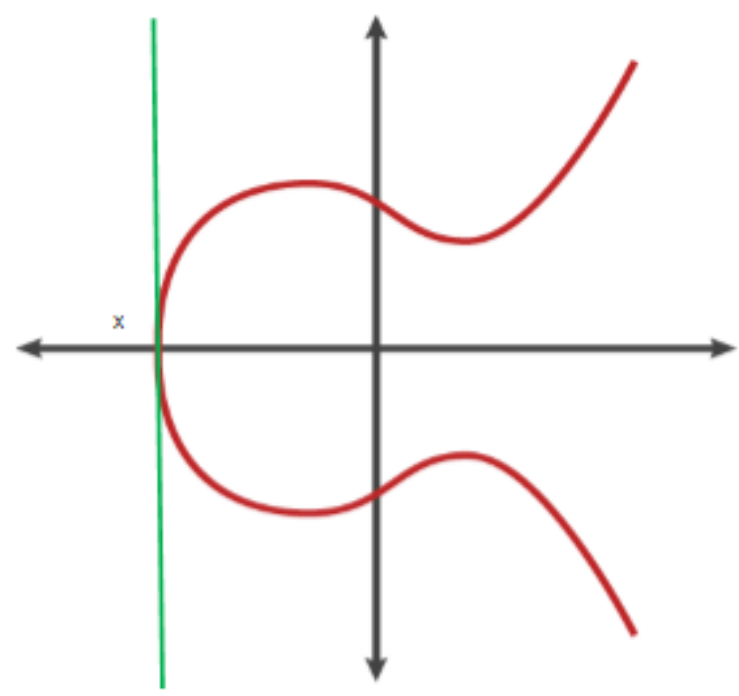

Figure 4. Graph of curves $Y+Y+0=0$.

The smart contract is translated into machine-level byte code where each byte defines each process, and then added as an EVM-1 transaction to the blockchain. A miner picks it up, and confirms Block-1. When a user sends the query through the web interface, the EVM-2 queries and embeds the web-based data into Transaction tx and deploys it to the blockchain. In Block-2 the transaction tx status is changed. When node 3 decides to test the states that are stored in the contract, then it must synchronize the changes up to at least Block -2 to see the changes that transaction made. 


\section{Blockchain Based Smart Contract system to curb the transmission of COVID-19}

A smart contract, based on blockchain technology, may be built and would have all the conditions from handling various permissions to accessing data as seen in Figure 5 and it can be seen that a variety of stakeholders are participating in this scheme performing different activities. It would help to create stronger physician-patient experiences. The rules regulating data authorisation are integrated into smart contracts. It can also help monitor all actions from their origin to their surrender, with unique Id. Alongside all the roles and procedures, a smart contract with various interactions has been developed and clarified well integrated in the smart contracts. Figure 5 shows the function of smart contracts with Ethereum, where for simplification the mining process is left out. There will be no need for a centralized body to oversee and authorize the process as it can be handled directly via the smart contract that greatly reduces the management process administration costs.

The computer transactions are registered with private key (patient or physician) of the owner. The system's block content reflects data ownership and access permissions exchanged by members of a private peer-to-peer network. Blockchain technology supports the use of smart contracts which allow us to automate and monitor certain state transitions (such as a change of access rights or the rise of a new process record). We record patient-doctor relationships on an Ethereum blockchain through smart contracts which combine a medical record with viewing rights and data retrieval directions (effectively data pointers) for external server operation to ensure against manipulation, we include a cryptographic hash of the record on the blockchain to ensure data security. This network will be connected to the local and international databases to ensure adequate monitoring and containment of the infection.

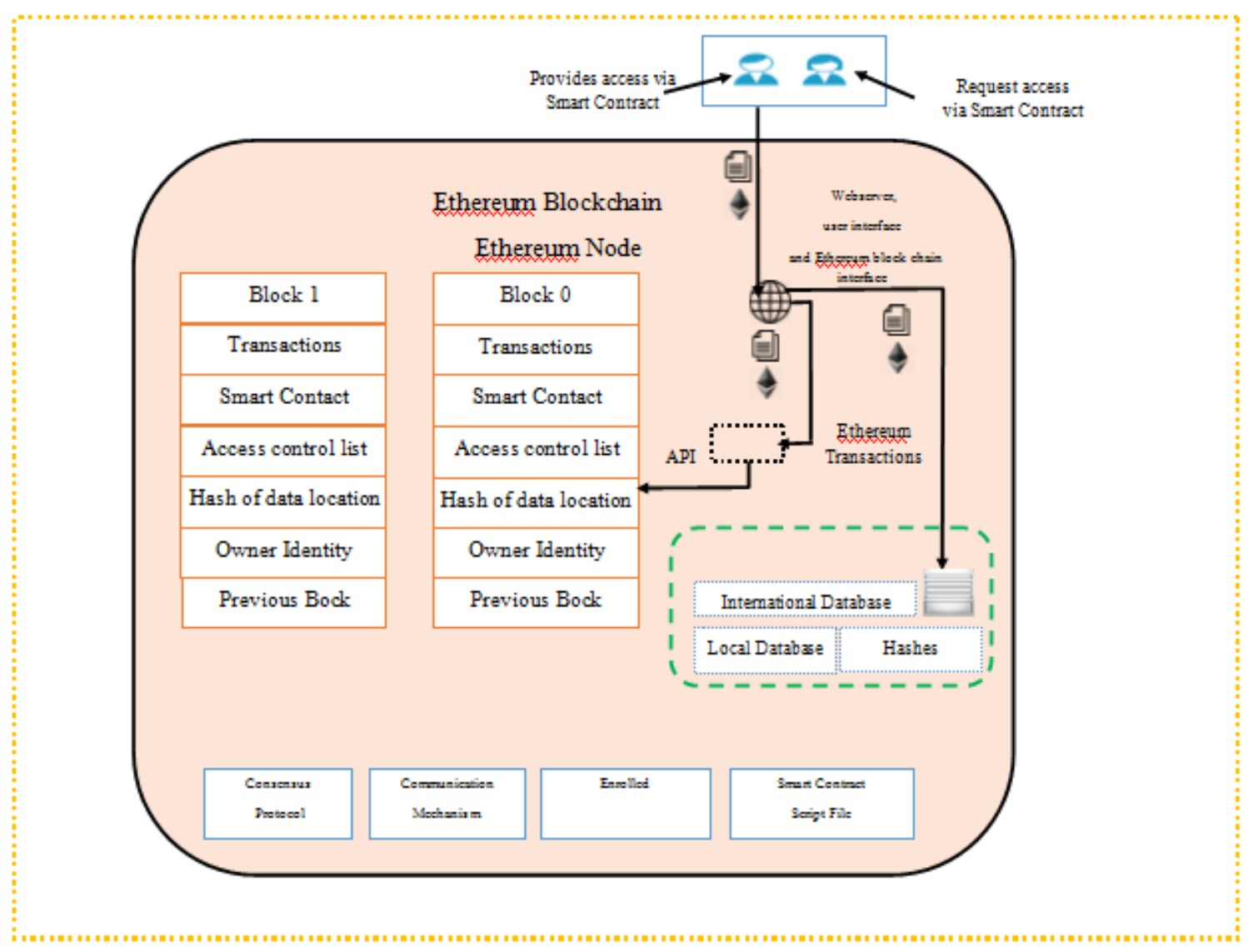

Figure 5. System Design and workflow with smart contract controlled access for COVID-19.

Health researchers need extensive data sets to advance disease awareness, speed up scientific discovery, rapidly monitor drug creation and design patient treatment strategies based on biology, lifecycle and environment. Through having patients of diverse ethnic and socio-economic backgrounds and from various geographic regions, Blockchain's shared data network will include a 
large variety of data set. It provides ideal knowledge for longitudinal studies because blockchain gathers data about a person's health over a lifetime.

A health care blockchain can expand health data processing to include data from groups of individuals presently under-served or not traditionally engaged in science. Blockchain's open data ecosystem makes it easier for "hard-to-reach" audiences to be involved, and more insightful for the general public to deliver results.

The will also facilitate the overcoming of administrative inefficiencies among patients, doctors and the healthcare organization. This system will assist in the rehabilitation, review and management of complex data and practices in the healthcare sector. The key objective is to share the information through smart blockchain contracts by enabling hospitals, physicians, emergency clinics and various partners to effectively access and exchange the therapeutic information of a patient amongst different stakeholders.

\section{Discussion}

WHO Director-General Dr Tedros Adhanom Ghebreyesus indicated: "About 3.4 percent of confirmed cases of COVID-19 have died globally. Seasonal flu, by contrast, typically kills much fewer than 1 per cent of those infected [34]. Originally, in a press conference on Wednesday, January 29 and again on February 10, the World Health Organization (WHO) listed 2 per cent as a mortality rate prediction. However, WHO stated on January 29 that this was a very early and preliminary estimation which may have changed. Surveillance has been rising, not just within China but also internationally, but it was said at the time: We do not even know exactly how many people have been infected ("When you think of how many people have died, you have to think at how many people have been infected and we don't know the amount right now. So it's early to write a figure on it." The only number actually known is how many of those who were registered to the WHO have died. According to the World Health Organisation, it is still very early to make any definitive claims about the overall mortality rate for the novel coronavirus.

Our proposed framework uses blockchain technology to build an iterative, scalable, stable, open and decentralized health-care ecosystem. This will encourage patients to openly and safely share their medical records with doctors, hospitals, research organizations and other stakeholders-all while retaining full control over the privacy of their medical data. It would fix many of the challenges of the existing healthcare system, including data siloing, incongruity with the legacy network, unstructured data processing difficulties, prohibitively high operating costs, lack of data protection and unaddressed privacy concerns. Our proposed blockchain-based approach may be modified to use other infectious diseases which could be intensified by the latest COVID-19 epidemic, in communitybased case finding.

\section{Conclusion}

Through blockchain technology, our smart contract-based healthcare management program has shown how the concepts of decentralization can be applied to large-scale data processing in medical environments and to streamline complex medical procedures. We demonstrate a revolutionary approach to the handling of medical records, offering auditability, interoperability and transparency through smart contracts. Built to document accessibility and granularity, this program enables the exchange of patient data and opportunities for medical researchers to support the system. From a medical perspective we have introduced a data management and sharing system focused on the requirements. It is possible to ensure the use of blockchain technology, privacy, protection, availability and a fine-grained control of patient data access. The ultimate goal of using blockchain as explained in this paper is to strengthen the processes of healthcare and thus patient outcomes to combat the infection (COVID-19). Blockchain can aid in many ways; minimizing transaction costs by using smart contracts that are embedded protocols for general purposes to simplify processes, eliminate administrative burdens and remove intermediaries. 
Funding: This research is supported by the Employment Based Postgraduate Program of the Irish Research Council (IRC) ) Project ID: EBPPG/2015/185 and partially funded under the SFI Strategic Partnership Program by Science Foundation Ireland (SFI) and FotoNation Ltd. (Xperi Corporation) Project ID: 13/SPP/I2868 on Next Generation Imaging for Smartphone and Embedded Platforms."

Funding: The author would like to express her sincere thanks and gratitude to Prof. Chris Dainty for his continuous support, advice and supervision at all stages of this research work. The author would also like to thank Prof. Peter Corcoran for his supervision and guidance throughout the period of this research.

Conflicts of Interest: The authors declare no conflict of interest.

\section{References:}

1. “WHO/Europe I Coronavirus disease (COVID-19) outbreak." [Online]. Available: http://www.euro.who.int/en/health-topics/health-emergencies/coronavirus-covid-19. [Accessed: 04-Apr2020].

2. Worldometer, “Coronavirus Cases," Worldometer, 2020. [Online]. Available: https://www.worldometers.info/coronavirus/coronavirus-cases/\#daily-cases. [Accessed: 04-Apr-2020].

3. A. Azaria, A. Ekblaw, T. Vieira, and A. Lippman, “MedRec: Using blockchain for medical data access and permission management," Proc. - 2016 2nd Int. Conf. Open Big Data, OBD 2016, pp. 25-30, 2016.

4. Jp. Genestier et al., "Blockchain for Consent Management in the eHealth Environment: A Nugget for Privacy and Security Challenges," J Int Soc Telemed eHealth, vol. 5, pp. 24-25, 2017.

5. D. Randall, P. Goel, and R. Abujamra, “Blockchain Applications and Use Cases in Health Information Technology," J. Heal. Med. Informatics, vol. 08, no. 03, pp. 8-11, 2017.

6. I. Radanović and R. Likić, “Opportunities for Use of Blockchain Technology in Medicine," Appl. Health Econ. Health Policy, vol. 16, no. 5, pp. 583-590, Oct. 2018.

7. J. Vora et al., “BHEEM: A Blockchain-Based Framework for Securing Electronic Health Records,” 2018 IEEE Globecom Work. GC Wkshps 2018 - Proc., pp. 1-6, 2019.

8. L. A. Linn and M. B. Koo, "Blockchain For Health Data and Its Potential Use in Health IT and Health Care Related Research," ONC/NIST Use Blockchain Healthc. Res. Work., pp. 1-10, 2016.

9. "Blockchain and Electronic Healthcare Records By: Nir Kshetri Kshetri, Nir (2018)." Blockchain and Electronic Healthcare Records ", IEEE," vol. 51, 2018.

10. X. Yue, H. Wang, D. Jin, M. Li, and W. Jiang, “Healthcare Data Gateways: Found Healthcare Intelligence on Blockchain with Novel Privacy Risk Control," J. Med. Syst., vol. 40, no. 10, pp. 1-8, Oct. 2016.

11. A. Petre, “Blockchain use cases in healthcare," LinkedIn Pulse, vol. 111, pp. 1-41, 2017.

12. R. Surya and G. C. P. Latha, "Blockchain: A panacea for healthcare cloud -based data security and privacy," Test Eng. Manag., vol. 82, no. February, pp. 6671-6676, 2020.

13. "Healthcare transaction validation via blockchain proof-of-work, systems and methods," May 2015.

14. M. A. Engelhardt, "Hitching Healthcare to the Chain: An Introduction to Blockchain Technology in the Healthcare Sector," Technol. Innov. Manag. Rev., vol. 7, no. 10, pp. 22-34, 2017.

15. B. Shen, J. Guo, and Y. Yang, “MedChain: Efficient healthcare data sharing via blockchain,” Appl. Sci., vol. 9, no. 6, 2019.

16. K. V. O. Rabah, “Challenges \& Opportunities for Blockchain Powered Healthcare Systems: A Review.” 2017. 
17. C. Agbo, Q. Mahmoud, and J. Eklund, “Blockchain Technology in Healthcare: A Systematic Review," Healthcare, vol. 7, no. 2, p. 56, 2019.

18. H. D. Zubaydi, Y. W. Chong, K. Ko, S. M. Hanshi, and S. Karuppayah, "A review on the role of blockchain technology in the healthcare domain," Electron., vol. 8, no. 6, pp. 1-29, 2019.

19. W. J. Gordon and C. Catalini, "Blockchain Technology for Healthcare: Facilitating the Transition to PatientDriven Interoperability," Comput. Struct. Biotechnol. J., vol. 16, pp. 224-230, 2018.

20. T. K. Mackey et al., "'Fit-for-purpose?' - Challenges and opportunities for applications of blockchain technology in the future of healthcare," BMC Med., vol. 17, no. 1, pp. 1-17, 2019.

21. M. N. Kamel Boulos, J. T. Wilson, and K. A. Clauson, “Geospatial blockchain: Promises, challenges, and scenarios in health and healthcare," International Journal of Health Geographics, vol. 17, no. 1. BioMed Central Ltd., 05-Jul-2018.

22. D. V. Dimitrov, “Blockchain applications for healthcare data management,” Healthc. Inform. Res., vol. 25, no. 1, pp. 51-56, 2019.

23. A. T. Litchfield, A. Khan, " A And Khan, and A. Khan, “CONF-IRM) 5-2019 Review of Issues in Healthcare Information Management Systems and Blockchain Solutions," vol. 1, 2019.

24. X. Liang, J. Zhao, S. Shetty, J. Liu, and D. Li, “Integrating blockchain for data sharing and collaboration in mobile healthcare applications," IEEE Int. Symp. Pers. Indoor Mob. Radio Commun. PIMRC, vol. 2017Octob, pp. 1-5, 2018.

25. M. Greenberger, "Block what? The unrealized potential of blockchain in healthcare," Nurs. Manage., vol. 50, no. 5, pp. 9-12, May 2019.

26. P. Zhang, M. A. Walker, J. White, D. C. Schmidt, and G. Lenz, “Metrics for assessing blockchain-based healthcare decentralized apps," 2017 IEEE 19th Int. Conf. e-Health Networking, Appl. Serv. Heal. 2017, vol. 2017-Decem, pp. 1-4, 2017.

27. M. Hölbl, M. Kompara, A. Kamišalić, and L. N. Zlatolas, “A systematic review of the use of blockchain in healthcare," Symmetry (Basel)., vol. 10, no. 10, 2018.

28. S. Khezr, M. Moniruzzaman, A. Yassine, and R. Benlamri, “Blockchain technology in healthcare: A comprehensive review and directions for future research,” Appl. Sci., vol. 9, no. 9, pp. 1-28, 2019.

29. T. McGhin, K. K. R. Choo, C. Z. Liu, and D. He, “Blockchain in healthcare applications: Research challenges and opportunities," J. Netw. Comput. Appl., vol. 135, no. September 2018, pp. 62-75, 2019.

30. T. Kumar, V. Ramani, I. Ahmad, A. Braeken, E. Harjula, and M. Ylianttila, “Blockchain utilization in healthcare: Key requirements and challenges," 2018 IEEE 20th Int. Conf. e-Health Networking, Appl. Serv. Heal. 2018, pp. 1-7, 2018.

31. “US20150332283A1 - Healthcare transaction validation via blockchain proof-of-work, systems and methods Google Patents." [Online]. Available: https://patents.google.com/patent/US20150332283A1/en. [Accessed: 25Mar-2020].

32. A. Khatoon, “A Blockchain-Based Smart Contract System for Healthcare Management," Electronics, vol. 9, no. 1, p. 94, Jan. 2020.

33. A. Khatoon, P. Verma, J. Southernwood, B. Massey, and P. Corcoran, "Blockchain in energy efficiency: Potential applications and benefits," Energies, vol. 12, no. 17, pp. 1-14, 2019.

34. “Coronavirus Update (Live): 1,141,425 Cases and 61,205 Deaths from COVID-19 Virus Outbreak Worldometer." [Online]. Available: https://www.worldometers.info/coronavirus/. [Accessed: 04-Apr-2020]. 